\section{Decisions, decisions...}

Notch proteins regulate cell fate decisions and inhibit differentiation in many developmental systems. Notch1 signalling has been previously associated with tumorigenesis, but now one study indicates that this receptor protein might have the opposite role in skin cells.

The role of Notch signalling in mammalian skin is not well understood, although some in vitro studies have indicated that Notch induces differentiation in this tissue. In the March issue of Nature Genetics, Nicolas et al. perform tissue-specific gene targeting of Notch 1 in the mouse epidermis and in the corneal epithelium. The authors made the surprising observation that disruption of Notch 1 promoted epidermal and corneal hyperplasia. Within as little as 8 months of birth, these mice developed skin tumours in various parts of the body. A total of $95 \%$ of mice older than 12 months developed highly vascularized tumours that were histologically

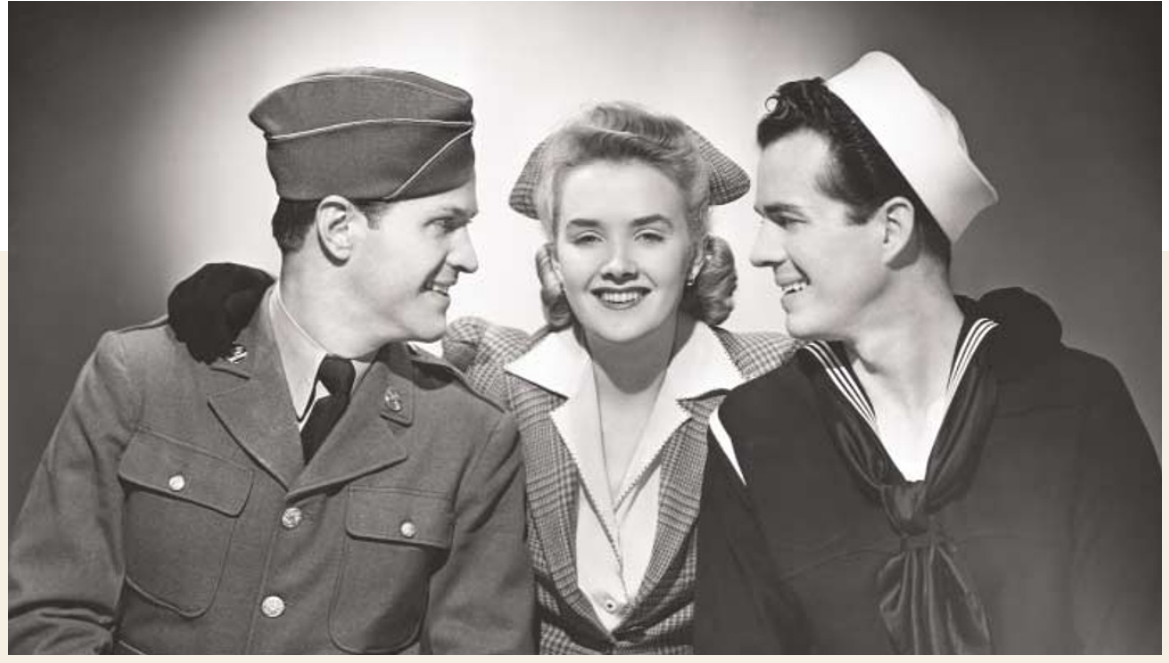

classified as basal-cell carcinomas. The mice were also more sensitive to chemical carcinogens.

These data were unexpected because, in other cell types, Notch1 is known to maintain proliferative cell populations in the undifferentiated state. In contrast with the common belief that Notch1 signalling is oncogenic, the findings of Nicolas et al. instead indicate that, in skin, Notch1 acts as a tumour suppressor. So what does Notch1 do in skin?

As basal-cell carcinomas are associated with activation of the Shh signalling pathway, Nicolas et al. looked to see if this pathway was activated in the tumours. They found that Notch 1 deficiency in skin and primary keratinocytes resulted in increased and sustained expression of Gli2 - a transcriptional target of Shh. So, Notch1 might function to repress Gli2 expression, and thereby promote keratinocyte differentiation.

The authors also found that Notch1 inhibits $\beta$-catenin signalling in differentiating keratinocytes, as well as restricting its expression to cells of the basal-cell layer of the epidermis. Loss of Notch1, along with upregulation of $\beta$-catenin and Gli2, can therefore promote a pro-proliferative, antidifferentiation state in skin cells.

Kristine Novak

6) References and links

ORIGINAL RESEARCH PAPER Nicolas, M. et al. Notch1

functions as a tumor suppressor in mouse skin. Nature Genet. 33, 416-421 (2003)

\title{
Getting under the skin
}

The transcriptional regulator NF- $\kappa B$ regulates several signalling pathways, including those that are involved in cell proliferation and apoptosis. In many tumours, activated NF- $\kappa B$ promotes proliferation and inhibits apoptosis, but reports have shown that inhibition of NF- $\kappa B$ actually causes increased proliferation in the skin. In the

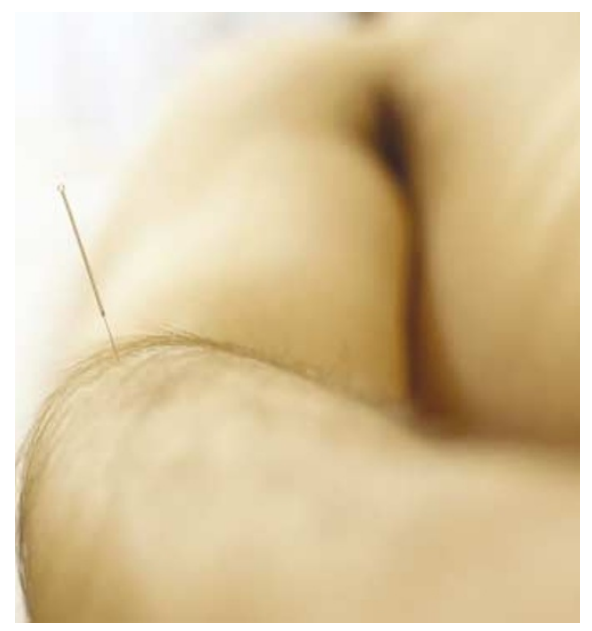

6 February issue of Nature, Maya Dajee et al. further explore the role of NF- $\mathrm{KB}$ in skin tumorigenesis.

The authors expressed activated oncogene HRAS either alone or with the NF- $\kappa B$ subunit p 65 or a stable mutant of IкB $\alpha$ - which causes $\mathrm{NF}-\mathrm{\kappa B}$ to remain inactive in the cytoplasm - in primary human keratinocytes and grafted these cells onto immunodeficient mice. RAS expression stimulates growth in most cell systems, but, in this model, it inhibited growth in the skin. Coexpression of RAS and p65 also inhibited proliferation. The co-expression of RAS and stabilized I $\mathrm{B} \mathrm{\alpha} \alpha$ allowed the cell to overcome growth arrest induced by activated RAS, leading to the formation of large squamous-cell carcinomas (SCCs). Tumours from patients with SCC had NF- $\kappa B$ in the cytoplasm and a subset also had increased expression of IKB $\alpha$ and RAS, supporting the relevance of control of RAS and NF- $\kappa B$ in some spontaneous human SCCs.

So, the functions of activated RAS and inactivated NF- $\kappa$ B oppose each other to cause carcinogenesis in the skin, but what other molecules are involved? The expression of integrin receptors - which interact with extracellular-matrix components, such as laminin, and trigger cell proliferation and migration - is associated with tumour-invasive potential. Dajee and colleagues showed that the SCCs with stabilized IКB $\alpha$ and activated RAS also expressed high levels of integrin $\alpha 6 \beta 4$ and laminin-5, and that blocking these molecules with specific antibodies inhibited tumour formation. When keratinocytes from patients with a blistering disease caused by mutations in integrin $\alpha 6 \beta 4$ and laminin-5 were transduced with stabilized IкB $\alpha$ and activated RAS, no tumours were formed, indicating that integrin and laminin also have a key role in SCC development.

These data highlight the problems of targeting NF- $\kappa B$ as a method of increasing apoptosis in tumours - use of such agents, which are in development, could actually promote tumorigenesis in the skin.

\section{Ezzie Hutchinson}

6) References and links

ORIGINAL RESEARCH PAPER Dajee, M. et al. NF-KB

blockade and oncogenic Ras trigger invasive human epidermal neoplasia. Nature 421, 639-643 (2003) FURTHER READING

Karin, M. et al. NF-kB in cancer: from innocent bystander to major culprit. Nature Rev. Cancer 2, 301-310 (2002) WEB SITE

WEB SITE
Paul A. Khavari's lab: http://cmgm.stanford.edu/khavari/ 